\title{
A comparison of clustering methods for biogeography with fossil datasets
}

Matthew J Vavrek

Cluster analysis is one of the most commonly used methods in palaeoecological studies, particularly in studies investigating biogeographic patterns. Although a number of different clustering methods are widely used, the approach and underlying assumptions of many of these methods are quite different. For example, methods may be hierarchical or nonhierarchical in their approaches, and may use Euclidean distance or non-Euclidean indices to cluster the data. In order to assess the effectiveness of the different clustering methods as compared to one another, a simulation was designed that could assess each method over a range of both cluster distinctiveness and sampling intensity. Additionally, a nonhierarchical, non-Euclidean, iterative clustering method implemented in the R Statistical Language is described. This method, Non-Euclidean Relational Clustering (NERC), creates distinct clusters by dividing the data set in order to maximize the average similarity within each cluster, identifying clusters in which each data point is on average more similar to those within its own group than to those in any other group. While all the methods performed well with clearly differentiated and well-sampled datasets, when data are less than ideal the linkage methods perform poorly compared to non-Euclidean based $k$-means and the NERC method. Based on this analysis, Unweighted Pair Group Method with Arithmetic Mean and neighbor joining methods are less reliable with incomplete datasets like those found in palaeobiological analyses, and the $k$-means and NERC methods should be used in their place. 


\section{A comparison of clustering}

2

5 Matthew J. Vavrek ${ }^{1}$

6

7

\section{Abstract}

${ }^{1}$ Royal Ontario Museum, Department of Natural History, 100 Queen's Park, Toronto, Ontario M5S 2C6, Canada

\section{fossil datasets}

Cluster analysis is one of the most commonly used methods in palaeoecological studies, particularly in studies investigating biogeographic patterns. Although a number of different clustering methods are widely used, the approach and underlying assumptions of many of these methods are quite different. For example, methods may be hierarchical or non-hierarchical in their approaches, and may use Euclidean distance or non-Euclidean indices to cluster the data. In order to assess the effectiveness of the different clustering methods as compared to one another, a simulation was designed that could assess each method over a range of both cluster distinctiveness and sampling intensity. Additionally, a non-hierarchical, non-Euclidean, iterative clustering method implemented in the R Statistical Language is described. This method, NonEuclidean Relational Clustering (NERC), creates distinct clusters by dividing the data set in order to maximize the average similarity within each cluster, identifying clusters in which each data point is on average more similar to those within its own group than to those in any other group. While all the methods performed well with clearly differentiated and well-sampled datasets, when data are less than ideal the linkage methods perform poorly compared to nonEuclidean based $k$-means and the NERC method. Based on this analysis, Unweighted Pair Group Method with Arithmetic Mean and neighbor joining methods are less reliable with incomplete 
26 datasets like those found in palaeobiological analyses, and the $k$-means and NERC methods

27 should be used in their place.

28

29 Keywords: Adjusted Rand Index, biogeography, cluster analysis, ecological similarity, 30 palaeoecology

\section{Introduction}

Clustering, defined as "a classificatory method which optimizes intra-group homogeneity" (Lance and Williams, 1967), is one of the most frequently used forms of multivariate analysis in palaeoecology (Hammer et al., 2001). One of the areas in which cluster analysis is commonly used is studying patterns of biogeography amongst species assemblages. Cluster analysis has been used in palaeoecological studies on groups as diverse as vertebrates (Shubin and Sues, 1991; Holtz, Jr. et al., 2004; Fröbisch, 2009; Gates et al., 2010; Noto and Grossman, 2010; Donohue et al., 2013), invertebrates (Schwimmer, 1975; Clapham and James, 2008), foraminifera (Collins, 1993) and plants (LePage et al., 2003), and assemblages spanning the Ediacaran (Clapham et al., 2003) to the Pleistocene (Wolfe, 2000). With the rise of large datasets of fossil species occurrences [e.g. Paleobiology Database, MioMAP (Carrasco et al. 2005), FAUNMAP (Graham and Lundelius, Jr. 2010), NOW (Fortelius 2015); see Uhen et al., 2013 for recent review] with hundreds or thousands of records, semi-automated methods such as clustering are becoming more and more necessary to find underlying patterns in these highly complex collections. As the use of cluster analysis in palaeobiology has steadily expanded, so too have the types of methods used. Although the underlying purpose of these methods is the same (i.e. to delimit different groups from one another), their approaches and assumptions are often quite different. For example, some cluster analysis methods (e.g. Unweighted Pair Group Method with Arithmetic Mean/UPGMA, neighbour-joining) use a hierarchical approach to grouping data (James and McCulloch, 1990; Shi, 1993).

Other common methods include partitioning techniques, such as $c$-means or $k$-means, which may try to optimize groups by minimizing relative distances based on a chosen index

54 (Hartigan and Wong, 1979). Although clustering methods may be widely used, their 
56 datasets used in palaeobiological studies. In order to examine the relative efficacy of these

57 different clustering methods with species occurrence data, a dataset where the "true" clustering 58 relationship is known is required. To generate multiple simulated datasets with established

59 clustering relationships, I created an R function which could create a species occurrence database

60 that could then be used to test the efficiency of the methods over a large number of trials.

In addition to the analysis of the various clustering methods commonly used, I also describe here an $\mathrm{R}$ function for a non-Euclidean, non-hierarchical clustering method termed here Non-Euclidean Relational Clustering (NERC), an iterative method that uses agglomerative clustering with post-clustering optimization. The efficacy of this function is tested in comparison to the more traditional methods.

Materials and Methods

\section{The NERC Function}

The NERC Function The algorithm's execution can be broken down into three distinct steps [after Lance and Williams (1967)]: the initialization of clusters; the allocation of new elements to a cluster; and finally an iterative reallocation process whereby the clusters are optimized. The first step, initialization of the clusters, begins by sampling a number of elements equal to the requested number of final clusters. Each of these selected samples is assigned randomly to a different initial cluster. In the second step, the function searches for the greatest similarity (smallest value in a dissimilarity matrix) between any unassigned sample and any assigned sample. The unassigned sample with the highest similarity is assigned to the same group as that which it shares the greatest similarity, similar to Single Linkage Clustering Analysis (Gower and Ross, 1969). This process then repeats, until all samples are assigned to a cluster. At the end of the second step, if any group has only one member the process restarts from the first step.

As a final step, an optimization of the clusters is performed. To begin, each individual sample within the entire set is assessed for its average similarity to every cluster. The similarity is based on the average pairwise distance from a sample to every member of a cluster (excluding the sample itself in the case of the cluster it had been assigned to). If a sample has a greater 
85 similarity to another cluster other than the one it has been assigned to, the optimization routine 86 will reassign the sample to the cluster that it had the most similarity to. If more than one sample 87 is in a suboptimal cluster, only one sample, chosen at random, will be reassigned at a time. After 88 a sample has been reassigned, the average pairwise distances will be calculated again before 89 another sample is reassigned (if necessary). If all the samples are in the cluster with which they have the greatest average similarity then the cycle is complete. At present, an upper limit of 1000 reassignments has been set so as to avoid an infinite loop if there is no solution where every sample is in its optimal grouping. The process will find a local, but not necessarily global, optimum by minimizing the overall dissimilarity within clusters. Because the method is heuristic in nature, it is best to repeat the clustering process many times.

\section{Implementation of NERC}

The R Statistical Language (R Development Core Team, 2012) was used to implement the NERC function. The R Language is cross platform, Open Source and free to use, and is widely used in statistical research, making it easy to extend with new functions and packages. The package fossil (Vavrek, 2011) with all of the functions discussed in this paper is available through the Comprehensive R Archive Network (CRAN) at http://cran.rproject.org/web/packages/fossil/. All data analysis and figure creation was done using R v3.2.1 on a Mac OS X 10.10 system. For a full copy of the R code used in the calculations and figures, please consult the Supplementary Materials.

The R implementation of the NERC function has one required and three optional arguments, and takes the form:

$$
\text { rclust (dist, clusters }=2 \text {, rand }=1000 \text {, counter }=\text { FALSE) }
$$

110 The only required argument is a distance or dissimilarity matrix (the dist argument), either as a

111 full matrix or lower triangle. The first optional argument (clusters) is the number of groups to be 112 created. The number of groups used must be a positive integer equal to or greater than 2 but no 113 greater than $1 / 2$ the total number of samples. The minimum value represents the smallest number 114 of clusters without placing all samples within one group, and the maximum value prevents 
115 clusters of one. The default value for the number of clusters is set to 2 . The second optional

116 argument gives the number of times the clustering process should be run. Because the method

117 should be run many times to have a better chance of finding the global optimal solution, this

118 option has a default value of 1000 . The last optional argument (counter) specifies whether to

119 print the current run. Note that at this point the R function returns only the result with the

120 smallest average within group distances overall.

\section{Data Simulation and Comparisons}

In order to test the efficacy of NERC in comparison to several other cluster methods, I

124 also created a simple function to simulate a species abundance data set. This function, called

$125 \operatorname{sim} .0 c c()$, creates a matrix of sites (columns) and species (rows) with a known clustering

126 solution. The number of species, localities, regions (clusters), sample size and proportion of

127 regional endemicity can all be adjusted. Each specific 'region' in the simulated set contains a

128 number of 'cosmopolitan' species that are found in every region, as well as 'endemic' species

129 that are found in only that particular region. To obtain a sample for a single locality, a

130 randomized log-normal distribution is applied to the total possible species pool for a given

131 region; the parameters are set so that any given locality will have several abundant species, a

132 large number of less common species, and some species which are not present. A log-normal

133 distribution was used as it is one of the most common species abundance distributions found in

134 empirical samples of modern habitats (Preston 1962; Gaston and Blackburn 2000; Magurran

135 2004). For every sample a new randomized log-normal distribution was created from the parent

136 region species pool. The average number of specimens can be varied to simulate different

137 sampling intensities. The full $\mathrm{R}$ code for the function can be found within the fossil package.

138 The simulated data was clustered using 6 different combinations of methods and input

139 matrices: single linkage, complete linkage, UPGMA, $k$-means on a db-RDA ordination using

140 both Euclidean and a non-Euclidean distance measure, and NERC. For those methods that

141 provide hierarchical clusters, discrete clusters were made using the cutree function. The db-RDA

142 ordination was performed using the capscale function in the vegan (Oksanen et al., 2011)

143 package.

144 Most functions used require a distance matrix as input, rather than raw species values. In 
145 order to convert the occurrence matrices to dissimilarity matrices, the ecol.dist() function was

146 used, with the Sørensen (sometimes called Dice) dissimilarity index used to calculate pairwise

147 dissimilarities. The Sørensen dissimilarity index was used because it is one of the most

148 commonly used indicices, and is regarded as one of the most effective presence/absence

149 dissimilarity measures (Southwood and Henderson 2000; Magurran 2004). Although the

$150 \operatorname{sim} .0 c c()$ function did create abundance-based occurrence matrices, the use of the Sørensen

151 dissimilarity index is presence/absence based, in effect converting the data. Although discarding

152 abundance data is not generally recommended in actual analyses, presence/absence data is

153 typically more common in palaeontological datasets, so using the Sorenson dissimilarity index

154 created a more realistic scenario.

155 The six methods were tested to see how well they performed both with varying levels of

156 endemicity (or differentiation between clusters; Fig. 1) as well as with varying levels of sampling

157 intensity. A simulated occurrence matrix was created 1000 times for each level of differentiation

158 or sampling intensity, and then clustered to obtain averaged performance values for all five

159 clustering methods. Each of the simulations consisted of 30 samples from 3 different endemic

160 regions, for a total of 90 samples to be used in the cluster analysis. Because of the parallel nature

161 of this simulation, the multicore (Urbanek, 2011), foreach (Revolution Analytics, 2011b), and

162 doMC (Revolution Analytics, 2011a) parallel computing packages for R were also used. The

163 visualization of cluster distinctiveness in Fig. 1 was created using the NMDS function provided

164 by the ecodist package (Goslee and Urban, 2007).

165 For the simulated biogeographic datasets, the "true" clustering was known, and so the

166 results of each clustering method could be compared to this a priori grouping. The Rand Index

167 (Rand, 1971; Hubert and Arabie, 1985) is method to compare two clustering outcomes and

168 calculates an index of similarity, with a value of 1 being a perfect match. The original formula

169 for this index, however, had a lower bound that fluctuated, depending on group sizes and

170 numbers (Hubert and Arabie, 1985). A modification of this original formula, given by Hubert

171 and Arabie (1985), scaled the value so that the greatest mathematically possible difference would

172 always be 0 , with the upper bound still set to 1 . This modification is referred to as the Adjusted

173 Rand Index (ARI). In the fossil package, both functions are provided, although only the ARI is

174 used to calculate the effectiveness of the clustering methods in this paper. 


\section{Results}

177 Overall, the NERC and non-Euclidean $k$-means methods were the most effective at 178 recovering the original groupings across the different levels of regional endemicity (Fig. 2), with 179 the NERC slightly outperforming the non-Euclidean $k$-means. Using a Euclidean distance metric 180 for the $k$-means method, even when the rest of the method and dataset are kept the same, led to a 181 notable reduction in performance. Complete linkage and UPGMA were readily able to recover 182 the correct clusters when the groups were relatively distinct. However, when the simulated 183 clusters were less distinct their effectiveness quickly declined. Single linkage clustering was least 184 effective and, produced unreliable results even at levels where all the other methods easily found 185 the proper clustering arrangement.

186 For the differing levels of sampling intensity (Fig. 3), the NERC method and non187 Euclidean $k$-means methods were again the most effective at recovering an accurate signal, 188 although in this instance the $k$-means was slightly more effective. Overall, complete linkage and 189 UPGMA gave accurate results when sampling intensity was high, but their performance was 190 very poor with sparsely sampled data. Single linkage was again the least effective of all the 191 methods tested.

\section{Discussion}

All cluster methods performed well when clusters were very distinct and sampling intensity was high. However, in cases where biogeographic clusters were less distinct or sampling was poor, the $\mathrm{db}-\mathrm{RDA} / k$-means and the NERC methods were best able to recover the original clusters compared to the other tested clustering methods. Among the other clustering methods, single linkage performed the poorest of any of the methods. The notably poor performance of the single linkage method was likely the result of individual samples that were extremely distant from all others placed at the base of the tree, and because I applied a strict tree cutting method with the hierarchical methods to obtain discrete clusters, the tree cutting method then identified this single distant sample as an individual cluster. However, the treatment of outliers is challenging in all clustering approaches, and their exclusion may not be possible or desirable. A similar situation, where outliers have an undue influence on group composition, is 
205 likely why complete linkage and UPGMA are also less effective than $k$-means or NERC.

206 These hierarchical methods are well suited to applications such as phenetic analyses or

207 phylogenetics, where a single ancestor (theoretically) gives rise to multiple descendants.

208 However, this one-to-many structure often translates poorly to species occurrence data sets like

209 those commonly used in biogeographic studies, where individual lineages may be operating in

210 parallel and independently (Brown, 1999). Individual species may originate in different locations

211 and disperse by various methods to new regions (Brown, 1999), leading to a more reticulate,

212 many-to-many relationship. In this case, a method that does not enforce a hierarchy may better

213 represent the relationships present.

214 Further, species occurrence data is typically non-Euclidean in nature. Whereas all the

215 cells in a phylogenetic data matrix represent a directly observed value, in a species occurrence

216 matrix any cell that has a zero value may be due to either the species not occurring in that area or

217 incomplete sampling, two possibilities that may be indistinguishable from one another. To deal

218 with incomplete sampling, most species occurrence data sets are converted into a distance

219 matrix, where the species composition of each sample is compared to every other sample using

220 an index of similarity (or dissimilarity); yet, while most of these measures provide some measure

221 of distance, these distances are not necessarily Euclidean (Gower and Legendre, 1986). The

222 benefit of using non-Euclidean measures over Euclidean distances is readily observable in this

223 study, with the non-Euclidean based $k$-means outperforming the Euclidean based $k$-means.

224 Although for this study, the Sørensen dissimilarity index was used, the choice of which

225 non-Euclidean dissimilarity index to use is not necessarily straightforward (e.g. Shi 1993;

226 Magurran 2004; Alroy 2015). By some counts, dozens of different dissimilarity indices have

227 been proposed in the literature (Hubálek 1982; Pielou 1984; Shi 1993), although only a handful

228 of these have entered into common use (Magurran 2004). While alternative methods, such as a

229 recent modification to the Forbes metric (Alroy 2015), have been proposed as replacements to

230 more traditional dissimilarity metrics, the choice of measure is a separate question to the issue in

231 the present study. Although using other dissimilarity measures may have changed the individual

232 effectiveness of the different clustering methods, the relative performance of the clustering

233 methods to each other is unlikely to change, as even with different measures the problems of

234 outliers and hierarchical/non-hierarchical methods would persist.

235 Both poor differentiation between clusters and inadequate sampling are common 
236 problems with palaeobiological data. No method is entirely immune to either of these issues, but

237 overall, based on these simulations, $k$-means and NERC give more reliable and accurate results

238 when data are less than robust. Using these methods still does make one strong assumption about

239 the underlying data - namely, that true divisions within the data exist. Unfortunately, with the

240 often muddled and noisy nature of biogeographic data, this assumption is also the hardest to

241 objectively determine.

\section{Acknowledgments}

243 I would like to thank Caleb M. Brown, Nicolás E. Campione, Luke B. Harrison, Pat A. Holroyd

244 and Brian D. Rankin for their critical feedback which improved the quality of this paper, and

245 Callum G. Vavrek and Ada E. Vavrek for their assistance in editing the manuscript. 
247 References

248 Alroy, J. (2015). A new twist on a very old binary similarity coefficient. Ecology, 96: 575-586. doi: 10.1890/14-0471.1.

250 Brown, J. H. (1999). Macroecology: Progress and Prospect. Oikos, 87(1):3-14.

251 Carrasco, M. A., Kraatz, B. P., Davis, E. B., and Barnosky, A. D. (2005). Miocene Mammal

252

253

254

255

256

257

258

259

260

261

262

263

264

265

266

267

268

269

270

271

272

273

274

275

276

Mapping Project (MIOMAP). University of California Museum of Paleontology, http://www.ucmp.berkeley.edu/miomap/

Clapham, M. E. and James, N. P. (2008). Paleoecology Of Early-Middle Permian Marine Communities In Eastern Australia: Response To Global Climate Change In the Aftermath Of the Late Paleozoic Ice Age. Palaios, 23(11):738-750.

Clapham, M. E., Narbonne, G. M., and Gehling, J. G. (2003). Paleoecology of the oldest known animal communities: Ediacaran assemblages at Mistaken Point, Newfoundland. Paleobiology, 29(4):527-544.

Collins, L. S. (1993). Neogene Paleoenvironments of the Bocas del Toro Basin, Panama. Journal of Paleontology, 67(5):699-710.

Donohue, S. L., Wilson, G. P., and Breithaupt, B. H. (2013). Multituberculates of the Black Butte Station local fauna (Lance Formation, southwestern Wyoming), with implications for compositional differences among mammalian. Journal of Vertebrate Paleontology, 33(May):677-695.

Fortelius, M. (coordinator). (2015). New and Old Worlds Database of Fossil Mammals (NOW). University of Helsinki. http://www.helsinki.fi/science/now/.

Fröbisch, J. (2009). Composition and similarity of global anomodont-bearing tetrapod faunas. Earth-Science Reviews, 95(3-4):119-157.

Gaston, K. J., and Blackburn, T. M. (2000). Pattern and Process in Macroecology. Blackwell Science, Ltd. doi: 10.1002/9780470999592.

Gates, T. A., Sampson, S. D., Zanno, L. E., Roberts, E. M., Eaton, J. G., Nydam, R. L., Hutchison, J. H., Smith, J. A., Loewen, M. A., and Getty, M. A. (2010). Biogeography of terrestrial and freshwater vertebrates from the late Cretaceous (Campanian) Western Interior of North America. Palaeogeography, Palaeoclimatology, Palaeoecology, 291(34):371-387. 
277 Goslee, S. and Urban, D. (2007). The ecodist package for dissimilarity-based analysis of 278 ecological data. Journal of Statistical Software, 22(7):1-19.

279 Gower, J. C. and Legendre, P. (1986). Metric and Euclidean properties of dissimilarity 280 coefficients. Journal of Classification, 3(1):5-48.

281 Gower, J. C. and Ross, G. J. S. (1969). Minimum Spanning Trees and Single Linkage Cluster 282 283 284 285 286 64.

Graham, R. W., and Lundelius, Jr., E.L. (2010). FAUNMAP II: New data for North America with a temporal extension for the Blancan, Irvingtonian and early Rancholabrean. FAUNMAP II Database, http://www.ucmp.berkeley.edu/faunmap/index.html

Hammer, Ø., Harper, D. A. T., and Ryan, P. D. (2001). PAST: paleontological statistics software package for education and data analysis. Palaeontologia Electronica, 4(1):9.

Hartigan, J. A. and Wong, M. A. (1979). A $k$-means clustering algorithm. Applied Statistics, 28(1):100- 108 .

Holtz, Jr., T. R., Chapman, R. E., and Lamanna, M. C. (2004). Mesozoic Biogeography of Dinosauria. In: Weishampel, D. B.,Dodson, P. and Osmólska, H., eds. The Dinosauria, Second Edition. University of California Press, 627-642.

Hubálek, Z. (1982). Coefficients of association and similarity, based on binary (presenceabsence) data: an evaluation. Biological Reviews, 57:669-689.

Hubert, L. and Arabie, P. (1985). Comparing partitions. Journal of Classification, 2(1):193-218.

James, F. C. and McCulloch, C. E. (1990). Multivariate analysis in ecology and systematics: panacea or Pandora's Box? Annual Review of Ecology and Systematics, 21(1):129-166.

Lance, G. N. and Williams, W. T. (1967). A general theory of classificatory sorting strategies: II. Clustering systems. The Computer Journal, 10(3):271-277.

LePage, B. A., Beauchamp, B., Pfefferkorn, H. W., and Utting, J. (2003). Late Early Permian plant fossils from the Canadian High Arctic: a rare paleoenvironmental/climatic window in northwest Pangea. Palaeogeography, Palaeoclimatology, Palaeoecology, 191(3-4):345372.

Magurran, A. E. (2004). Measuring Biological Diversity. Blackwell, Oxford.

Noto, C. R. and Grossman, A. (2010). Broad-scale patterns of Late Jurassic dinosaur paleoecology. PLOS ONE, 5(9):e12553. 
308 Oksanen, J., Blanchet, F. G., Kindt, R., Legendre, P., O’Hara, R. G., Simpson, G. L., Solymos, P., Stevens, M. H. H., and Wagner, H. (2011). vegan: Community Ecology Package.

Pielou, E. C. (1984). The interpretation of ecological data. Wiley, New York.

311 Preston, F. W. (1962). The canonical distribution of commonness and rarity: Part I. Ecology, 43: 185-215. doi: 10.2307/1931976.

313 R Development Core Team (2012). R: A Language and Environment for Statistical Computing.

314 R Foundation for Statistical Computing, Vienna, Austria. http://www.R-project.org/.

315 Rand, W. M. (1971). Objective criteria for the evaluation of clustering methods. Journal of the 316 American Statistical Association, 66(336):846-850.

317 Revolution Analytics (2011a). doMC: Foreach parallel adaptor for the multicore package.

318 Revolution Analytics (2011b). foreach: Foreach looping construct for R.

319 Schwimmer, D. R. (1975). Quantitative taxonomy and biostratigraphy of Middle Cambrian 320 trilobites from Montana and Wyoming. Journal of the International Association for 321 Mathematical Geology, 7(2):149-166.

322 Shi, G. R. (1993). Multivariate data analysis in palaeoecology and palaeobiogeography - a 323 review. Palaeogeography, Palaeoclimatology, Palaeoecology, 105(3-4):199-234.

324 Shubin, N. H. and Sues, H.-D. (1991). Biogeography of Early Mesozoic continental tetrapods:

325 Patterns and implications. Paleobiology, 17(3):214-230.

326 Uhen, M. D., Barnosky, A. D., Bills, B., Blois, J., Carrano, M. T., Carrasco, M. A., Erickson, G. M., Eronen, J. T., Fortelius, M., Graham, R. W., Grimm, E. C., O’Leary, M. A., Mast, A., Piel, W. H., Polly, P. D., and S äilä, L. K. (2013). From card catalogs to computers: databases in vertebrate paleontology. Journal of Vertebrate Paleontology, 33(1):13-28.

Urbanek, S. (2011). multicore: Parallel processing of R code on machines with multiple cores or CPUs.

Vavrek, M. J. (2011). fossil: palaeoecological and palaeogeographical analysis tools. Palaeontologia Electronica, 14(1):1T.

Wolfe, A. P. (2000). Paleoecology of a ¿90,000-year lacustrine sequence from Fog Lake, Baffin 336 Island, Arctic Canada. Quaternary Science Reviews, 19(17-18):1677-1699. 


\section{Figure captions}

338 Figure 1. Visualization of the changing endemicity of clusters (i.e. distinctiveness) and how it

339 alters the clustering of sites in an NMDS plot for the simulated biogeographic data sets. 'e' is the

340 proportion of all species that are endemic to only one biogeographic region. A higher proportion

341 of endemics results in more distinctive clusters, while a lower proportion of endemics results in

342 less distinctive clusters.

343

344 Figure 2. Response of various clustering methods to the distinctiveness of clusters as given by

345 the proportion of endemics (i.e. a higher endemicity creates more highly differentiated clusters).

346 The values for each method at any given level of endemicity is the average Adjusted Rand Index

347 comparing the known solution and the calculated solution over 1000 simulations.

348

349 Figure 3. Accuracy of various clustering methods in response to changing levels of sampling

350 intensity (coverage). Overall, as sampling intensity decreases (to the right), clustering becomes

351 less reliable. The values for each method at any given level of sampling is the average Adjusted

352 Rand Index comparing the known solution and the calculated solution over 1000 simulations. 


\section{Figure 1 (on next page)}

Variation in group distinctiveness for simulated data.

Visualization of the changing endemicity of clusters (i.e. distinctiveness) and how it alters the clustering of sites in an NMDS plot for the simulated biogeographic data sets. ' $e$ ' is the proportion of all species that are endemic to only one biogeographic region. A higher proportion of endemics results in more distinctive clusters, while a lower proportion of endemics results in less distinctive clusters. 


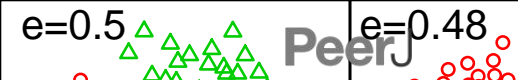

$\therefore 0$ H走

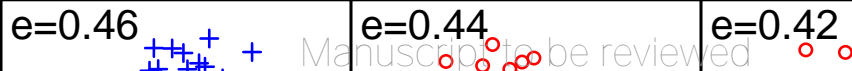




\section{Figure 2 (on next page)}

Comparison of cluster methods with varying group distinctiveness.

Response of various clustering methods to the distinctiveness of clusters as given by the proportion of endemics (i.e. a higher endemicity creates more highly differentiated clusters). The values for each method at any given level of endemicity is the average Adjusted Rand Index comparing the known solution and the calculated solution over 1000 simulations. 


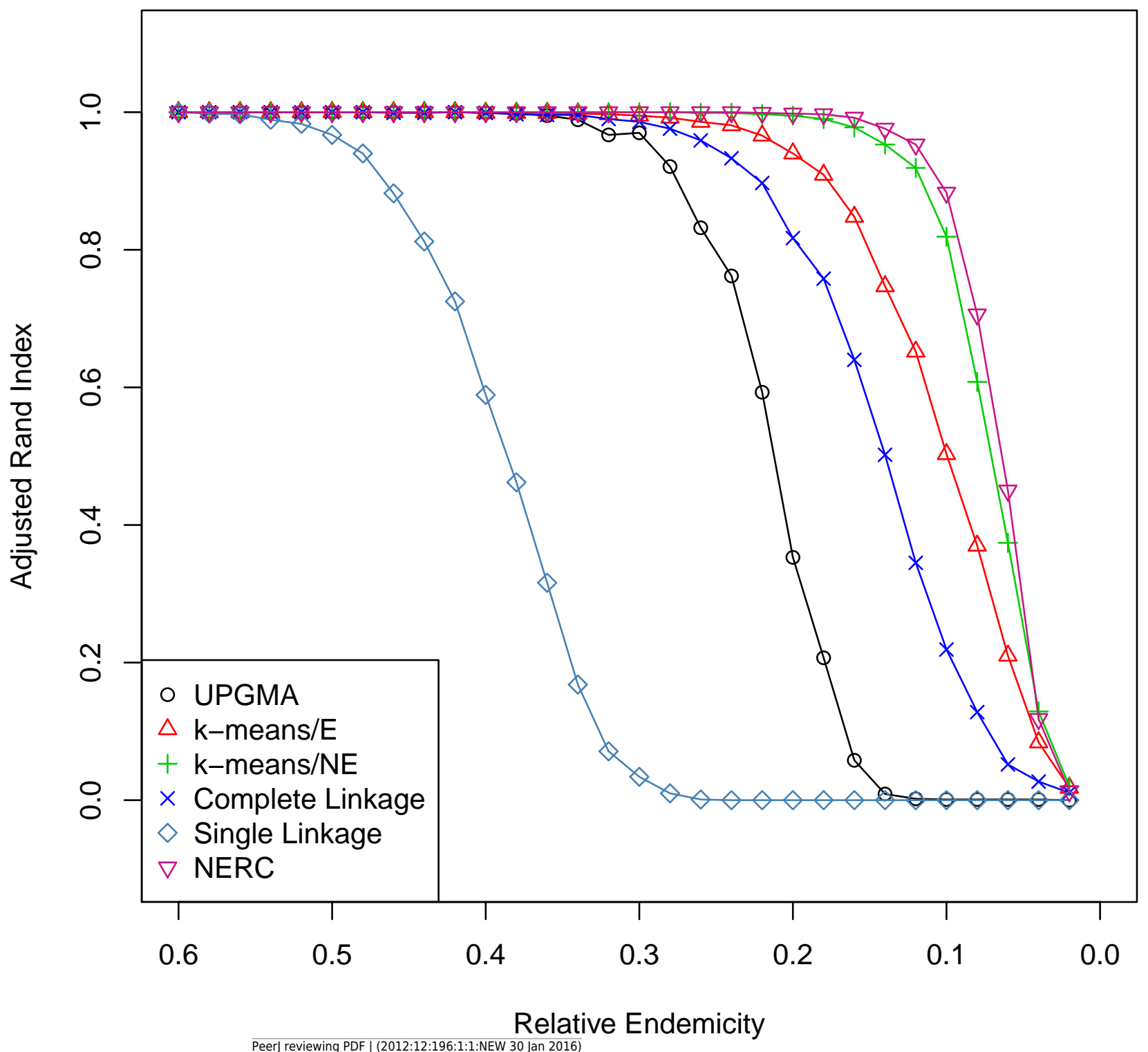


Figure 3 (on next page)

Comparison of cluster methods with varying sampling intensity.

Accuracy of various clustering methods in response to changing levels of sampling intensity (coverage). Overall, as sampling intensity decreases (to the right), clustering becomes less reliable. The values for each method at any given level of sampling is the average Adjusted Rand Index comparing the known solution and the calculated solution over 1000 simulations. 


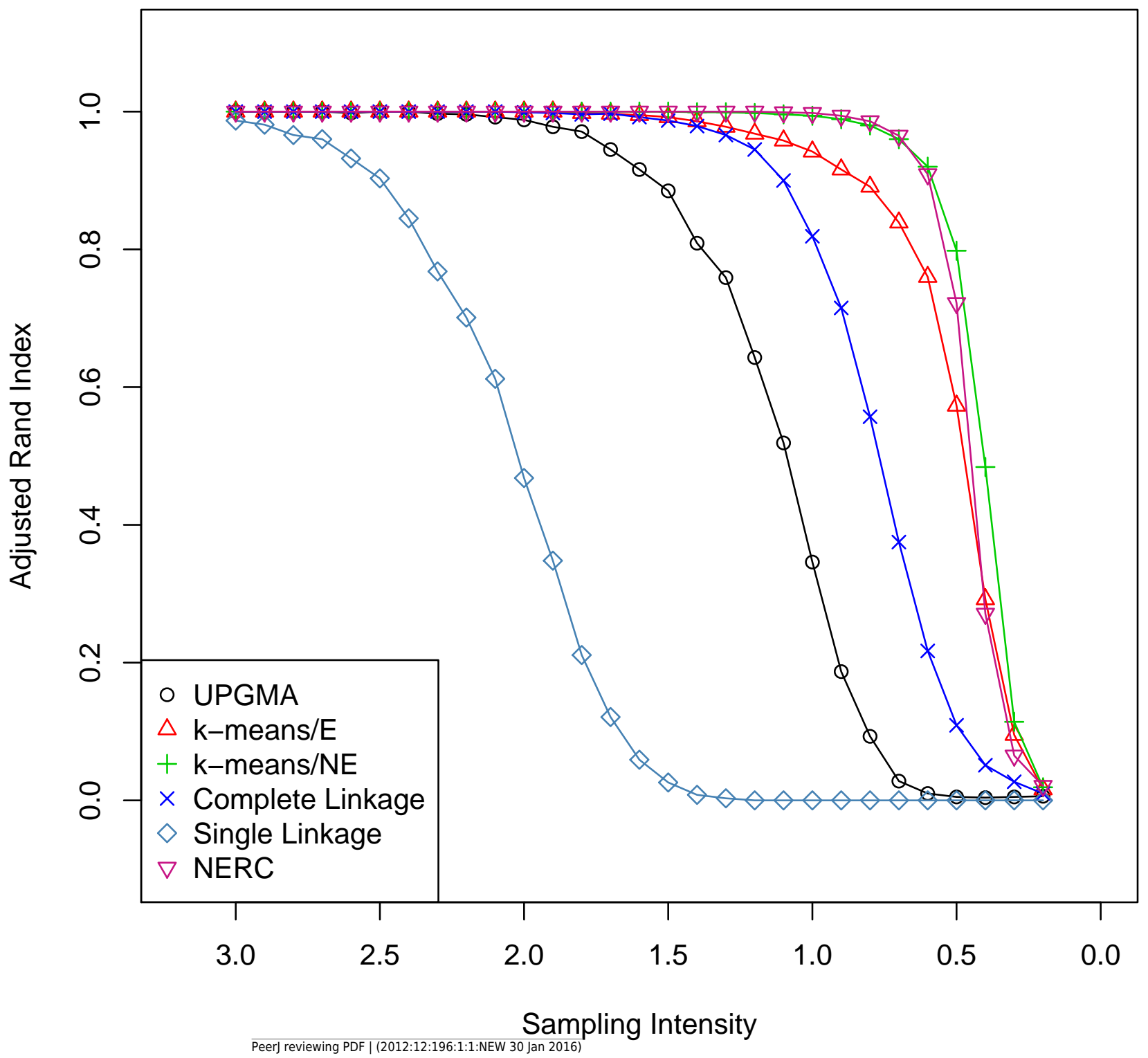

